\title{
4
}

\section{Determination of the Mass-Ratio Distribution, I: Single-lined Spectroscopic Binary Stars}

Accepted by: Astrophys. Space Sci.

\begin{abstract}
For single-lined spectroscopic binary stars (SBI), the mass ratio $q=M_{\mathrm{sec}} / M_{\text {prim }}$ is calculated from the mass function $f(m)$, which is determined from observations. For statistical investigations of the mass-ratio distribution, the term $\sin ^{3} i$, that remains in the cubic equation from which $q$ is solved, has to be dealt with.

This paper compares the common practise of taking an average value for $\sin ^{3} i$ to a deconvolution scheme that takes into account the precise (expected) behaviour of $P\left(\sin ^{3} i\right) d \sin ^{3} i$. The behaviour of $P\left(\sin ^{3} i\right) d \sin ^{3} i$ depends on how orbital planes of binary stars are oriented in space. For a random orientation of orbital planes, $P_{i}(i) d i=\sin i d i$. For the average value method, it is generally assumed that $P_{i}(i) d i=$ $(4 / \pi) \sin ^{2} i d i$.

For verification purposes, the deconvolution scheme is applied to an observed sample of double-lined spectroscopic binary systems (SBII), and to a synthetic sample of SBI systems, produced by a numerical model. In both cases, the scheme produces better results with the assumption that $P_{i}(i) d i=(4 / \pi) \sin ^{2} i d i$, rather than a purely random orientation of orbital planes. In the case of the synthetic sample of SBI systems, the deconvolution scheme does not produce better results than the method that assumes an average value for $\sin ^{3} i$.

Application of the deconvolution method to the double-lined spectroscopic binary systems in the Eighth Catalogue of the Orbital Elements of Spectroscopic Binary Stars, provides results which are compatible with the assumption that the orbital planes of these systems are oriented randomly in space.
\end{abstract}

Key words: spectroscopic binary stars, mass-ratio distribution, methods, deconvolution 


\subsection{Introduction}

This paper is part of a study of the mass-ratio distribution of spectroscopic binary stars (Hogeveen 1991, this thesis Chapter 3). It concentrates on how the mass-ratio distribution can be determined from catalogued data, such as those in the Eighth Catalogue of the Orbital Elements of Spectroscopic Binary Stars (Batten et al. 1989, referred to in this paper as DAO8).

Halbwachs (1987) has pointed out that the method which is generally applied to determine the mass ratio of single-lined spectroscopic binary systems (SBI) is not correct. He also points out the right way to handle the problem, and applies the new method to the sample of main-sequence spectroscopic binaries that is the subject of his paper. In her investigation of a sample of G- and K-type spectroscopic binaries (observed by Griffin), Trimble (1990) states that the method devised by Halbwachs does not yield results which are significantly different from those obtained by means of the traditional method.

In the present paper, the method devised by Halbwachs is refined and generalized. It is shown that the method provides better results with the assumption that orbital inclinations are distributed according to $P_{i}(i) d i=(4 / \pi) \sin ^{2} i d i$, than with the assumption of a purely random distribution $P_{i}(i) d i=\sin i d i$. For a synthetic sample of SBI systems the deconvolution method does not provide better results than the assumption of an average value $\left\langle\sin ^{3} i\right\rangle=0.679$, which is also based on $P_{i}(i) d i=(4 / \pi) \sin ^{2} i d i$.

\subsection{The method}

The mass ratio $q=M_{2} / M_{1}$ of single-lined spectroscopic binaries is calculated from the mass function:

$$
f(m)=\frac{M_{2}^{3} \sin ^{3} i}{\left(M_{1}+M_{2}\right)^{2}}=\frac{P K^{3}\left(1-e^{2}\right)^{3 / 2}}{2 \pi G} .
$$

Here $M_{1}$ and $M_{2}$ represent the masses of the primary and secondary components, $P$ is the orbital period, $K$ the radial velocity (semi) amplitude of the primary, $e$ the eccentricity of the orbit, $i$ the inclination of the orbit, and $G$ the gravitational constant.

The right hand term of Eq. (4.1) can be determined from observations. The mass ratio $q$ may then be obtained from the middle term of Eq. (4.1), by substituting $M_{2}=q M_{1}$. This yields a cubic equation, from which $q$ can be solved, provided $M_{1}$ is known:

$$
\frac{M_{1} \sin ^{3} i}{f(m)} q^{3}-(1+q)^{2}=0 .
$$

If no additional observations are available, the primary mass $M_{1}$ is generally determined from a spectral-type-mass relation (Trimble 1974).

In Eq. (4.2) the term $\sin ^{3} i$ remains. For statistical purposes, generally a mean or average value $\left\langle\sin ^{3} i\right\rangle=0.679$ is used (Trimble 1974, Staniucha 1979). When orbital planes of binary stars are assumed to be really oriented at random, the probability distribution of inclinations $i$ is $P_{i}(i) d i=\sin i d i$, resulting in $\left\langle\sin ^{3} i\right\rangle=0.589$. The 


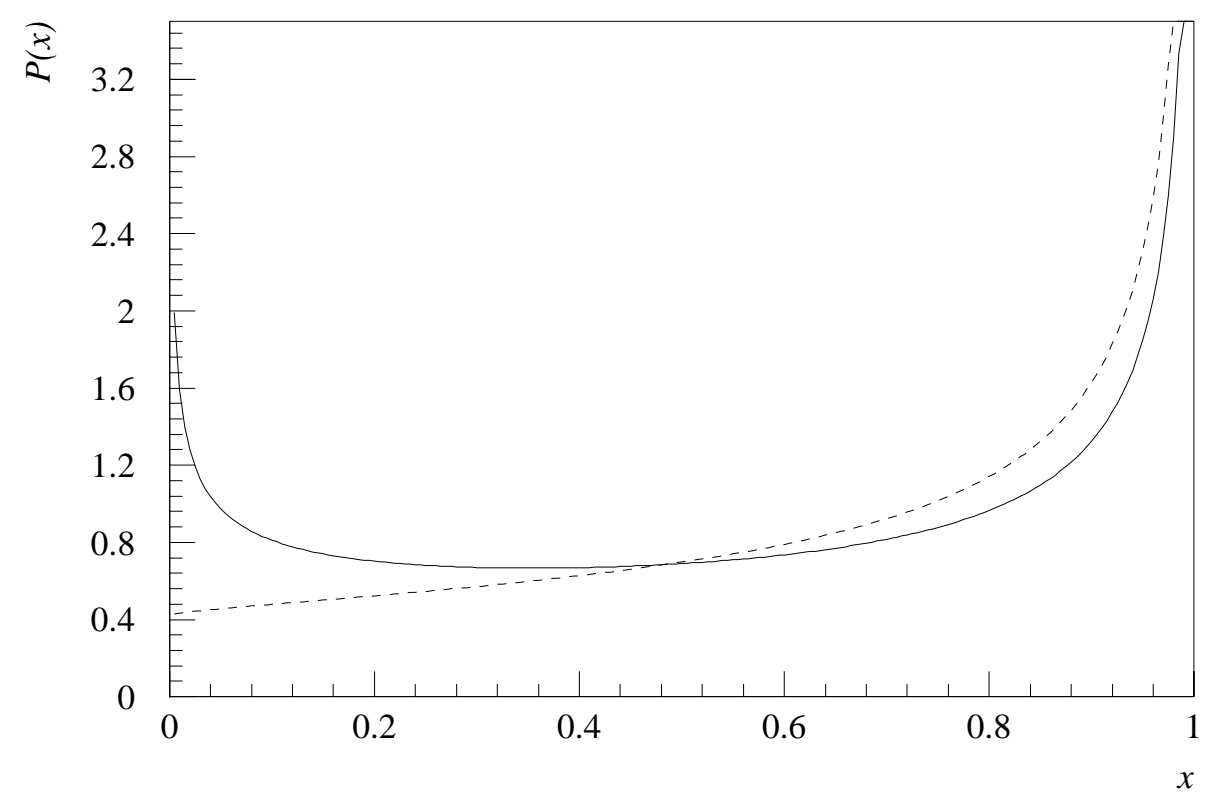

Figure 4.1: Plot of probability density $P(x) d x\left(x=\sin ^{3} i\right)$, assuming a random orientation of orbital planes in space $P_{i}(i) d i=\sin i d i$ (solid line), and a distribution $P_{i}(i) d i=(4 / \pi) \sin ^{2} i d i$ (dashed line) respectively. For the first case, $P(x) d x$ tends asymptotically to infinity for $x=0$ and $x=1$, in the latter case only for $x=1$. The normalization condition $\int P(x) d x=1$ is satisfied in both cases.

value $\left\langle\sin ^{3} i\right\rangle=0.679$ assumes $P(i)=(4 / \pi) \sin ^{2} i$, which should account for the fact that spectroscopic binary stars, with orbital planes which are observed almost face-on, are very hard to detect.

However, Halbwachs (1987) has shown that the most probable value of $\sin ^{3} i$ is not equal to its mean, but that the most probable values of $\sin ^{3} i$ are 0 and 1 . Figure 4.1 shows the theoretical probability distribution $P\left(\sin ^{3} i\right) d \sin ^{3} i$ of $\sin ^{3} i$, for $P_{i}(i) d i=$ $\sin i d i$ and $P_{i}(i) d i=(4 / \pi) \sin ^{2} i d i$ respectively. In the first case $P\left(\sin ^{3} i\right) d \sin ^{3} i$ tends asymptotically to infinity for $\sin ^{3} i \rightarrow 0$ and for $\sin ^{3} i \rightarrow 1$; in the latter case only for $\sin ^{3} i \rightarrow 1$. In both cases $\int P\left(\sin ^{3} i\right) d \sin ^{3} i$ is normalized to unity.

Following Halbwachs, we let $x=\sin ^{3} i$. Then $P\left(\sin ^{3} i\right) d \sin ^{3} i=P(x) d x$, and when $P_{i}(i) d i=\sin i d i$ it follows that

$$
P(x) d x=\frac{1}{3} x^{-1 / 3}\left(1-x^{2 / 3}\right)^{-1 / 2} d x .
$$

With $P_{i}(i) d i=(4 / \pi) \sin ^{2} i d i$ the expression becomes

$$
P(x) d x=\frac{4}{3 \pi}\left(1-x^{2 / 3}\right)^{-1 / 2} d x
$$

This is found by writing

$$
P_{i}(i) d i=P_{i}(i(x))\left|\frac{d i}{d x}\right| d x=P(x) d x
$$


where $P(x)=P_{i}(i(x))|d i / d x|$.

We will now investigate what the effect of the probability distribution $P\left(\sin ^{3} i\right)$, as it enters in Eq. (4.2), is on the observed mass-ratio distribution $\phi(q)$. First we will assume a purely random orientation of orbits in space, i.e., $P_{i}(i) d i=\sin i d i$.

For a system with known mass ratio $q^{\prime}$, and known inclination $i$, the observed values of $M_{1}$ and $f(m)$ may be expressed as:

$$
\frac{M_{1}}{f(m)}=\frac{\left(1+q^{\prime}\right)^{2}}{q^{\prime 3} \sin ^{3} i}
$$

But the value of $\sin ^{3} i$ is in general unknown to the observer of the system. In that case, a pseudo value $q^{*}$ of the mass ratio may be calculated by setting $\sin ^{3} i=1$ in $(4.2)$ :

$$
\frac{M_{1}}{f(m)} q^{* 3}-\left(1+q^{*}\right)^{2}=0
$$

The solution of this equation yields a pseudo mass ratio $q^{*} \leq q^{\prime}$.

When, for brevity, we write $B=\left(1+q^{\prime}\right)^{2} q^{-3}$ in (4.5), then we find by substitution in (4.2) that $\sin ^{3} i$ may be written as

$$
\sin ^{3} i=\frac{B q^{* 3}}{\left(1+q^{*}\right)^{2}} .
$$

$\sin ^{3} i$ has a value between 0 and 1 according to the distribution (4.3).

The probability $P_{q^{\prime}}\left(q^{*}\right)$, that a system with a real mass ratio $q^{\prime}$ is measured to have a pseudo mass ratio $q^{*}$ according to (4.6), is found by substituting (4.7) in (4.3):

$$
P(x) d x=P\left(x\left(q^{*}\right)\right)\left|\frac{d x}{d q^{*}}\right| d q=P_{q^{\prime}}\left(q^{*}\right) d q^{*},
$$

where we defined $P_{q^{\prime}}\left(q^{*}\right)=P\left(x\left(q^{*}\right)\right)\left|d x / d q^{*}\right|$. Substituting $x\left(q^{*}\right)=B q^{* 3}\left(1+q^{*}\right)^{-2}$, according to (4.7), in (4.3) yields

$$
P\left(x\left(q^{*}\right)\right)=\frac{\left(1+q^{*}\right)^{2 / 3}}{3 B^{1 / 3} q^{*}}\left\{1-\frac{B^{2 / 3} q^{* 2}}{\left(1+q^{*}\right)^{4 / 3}}\right\}^{-1 / 2}
$$

Thus Eq. (4.8) becomes

$$
P_{q^{\prime}}\left(q^{*}\right) d q^{*}=\frac{B^{2 / 3} q^{*}}{3\left(1+q^{*}\right)^{4 / 3}}\left\{1-\frac{B^{2 / 3} q^{* 2}}{\left(1+q^{*}\right)^{4 / 3}}\right\}^{-1 / 2}\left(3-\frac{2 q^{*}}{1+q^{*}}\right) d q^{*},
$$

with $B$ as defined above.

Figure 4.2 shows the behaviour of $P_{q^{\prime}}\left(q^{*}\right) d q^{*}$ for an arbitrary real mass-ratio value $q^{\prime}=0.8$. The distribution satisfies the normalization condition $\int P_{q^{\prime}}\left(q^{*}\right) d q^{*}=1$, and $P_{q^{\prime}}\left(q^{*}\right)$ asymptotically tends to infinity as $q^{*} \rightarrow q^{\prime}$.

When $x\left(q^{*}\right)=B q^{* 3}\left(1+q^{*}\right)^{-2}$ is substituted in (4.4), the redistribution $P_{q^{\prime}}\left(q^{*}\right) d q^{*}$ of systems with real mass ratios $q^{\prime}$ among pseudo mass ratios $q^{*}$ becomes

$$
P_{q^{\prime}}\left(q^{*}\right) d q^{*}=\frac{4 B}{3 \pi}\left(\frac{q^{*}}{1+q^{*}}\right)^{2}\left\{1-\frac{B^{2 / 3} q^{* 2}}{\left(1+q^{*}\right)^{4 / 3}}\right\}^{-1 / 2}\left(3-\frac{2 q^{*}}{1+q^{*}}\right) d q^{*} .
$$

The graph of this version of $P_{q^{\prime}}\left(q^{*}\right) d q^{*}$ has also been plotted in Fig. 4.2. 


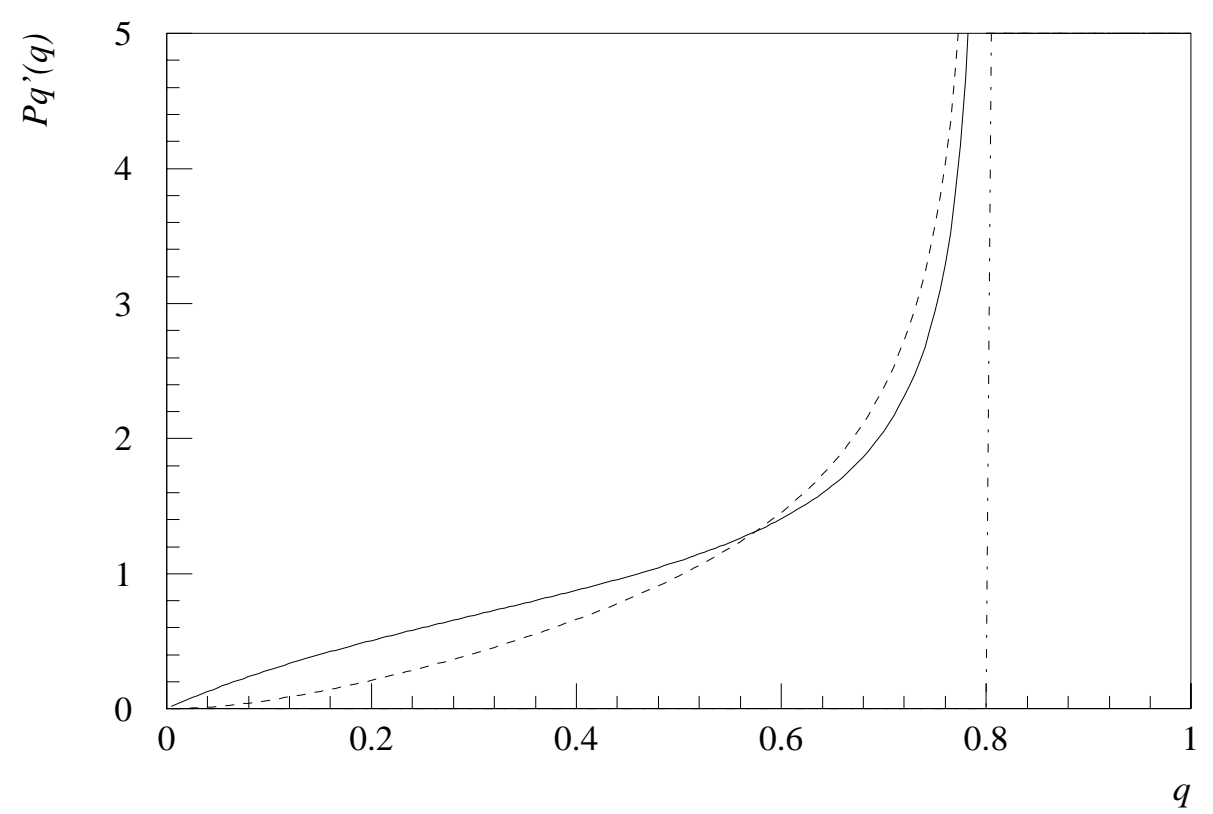

Figure 4.2: Solid line: plot of $P_{q^{\prime}}\left(q^{*}\right) d q^{*}$ according to Eq. (4.9). The graph shows the probability that a binary system with mass ratio $q^{\prime}=0.8$ is measured to have a pseudo mass ratio $q^{*}$, assuming a distribution $P\left(\sin ^{3} i\right)$ according to Eq. (4.3). Dashed line: plot of $P_{q^{\prime}}\left(q^{*}\right) d q^{*}$ according to Eq. (4.10) for an arbitrary $q^{\prime}=0.8$ and $P\left(\sin ^{3} i\right)$ according to Eq. (4.4). $\int P_{q^{\prime}}\left(q^{*}\right) d q^{*}=1 ; P_{q^{\prime}}\left(q^{*}\right)$ asymptotically tends to infinity for both distributions as $q^{*} \rightarrow q^{\prime}$.

\subsection{Deconvolution}

When the pseudo mass ratio of many SBI systems is calculated from their observed $M_{1}$ and $f(m)$ values according to Eq. (4.6), we may obtain a distribution of pseudo mass ratios $\psi\left(q^{*}\right)$. If we ignore other selection effects, the pseudo mass-ratio distribution $\psi\left(q^{*}\right)$ will be equal to the real mass-ratio distribution $\phi(q)$, smeared, or rather convolved with $P_{q^{\prime}}\left(q^{*}\right)$ :

$$
\psi\left(q^{*}\right)=\int_{q^{*}}^{1} \phi(q) P_{q}\left(q^{*}\right) d q .
$$

With the pseudo mass-ratio distribution $\psi\left(q^{*}\right)$ derived from observations, and expression (4.9) or (4.10) for $P_{q^{\prime}}\left(q^{*}\right)$, it should be possible to calculate the real mass-ratio distribution $\phi(q)$ from the deconvolution of Eq. (4.11).

Halbwachs (1987) did not calculate the deconvolution of Eq. (4.11), but he devised a numerical scheme to reconstruct $\phi(q)$ from $\psi\left(q^{*}\right)$, given the behaviour of $P_{q^{\prime}}\left(q^{*}\right)$. The scheme was suggested to him by a method, employed by Abt and Levy (1985), to determine the mass distribution of the secondary components of Am-type binaries.

In Halbwachs' scheme, the $q$-distribution is represented by a histogram, with bins of width $\Delta q=0.1$. The systems in the real $\phi(q)$ distribution are assumed to have only the discrete $q$-values $q_{1}=0.1, \ldots, q_{10}=1$. The scheme starts with assuming that all systems in bin 10 (bounded by $q^{*}=0.9$ and $q^{*}=1$ ) of the pseudo distribution $\psi\left(q^{*}\right)$, are due to a number of systems $N_{10}$ with real mass ratio $q_{10}=1$. When systems with 
$q=1$ are smeared with $P_{q^{\prime}=1}\left(q^{*}\right)$, then a fraction

$$
a_{i}=\int_{q_{i-1}}^{q_{i}} P_{q^{\prime}=1}\left(q^{*}\right) d q^{*}
$$

of them will be observed with a pseudo mass-ratio value $q^{*}$ between $q_{i-1}$ and $q_{i}$. If there are $n_{10}$ systems in bin 10 , then this requires $N_{10}=n_{10} / a_{10}$ systems with real mass ratio $q=1$.

The number of systems $a_{i} N_{10}$ in each bin $i$, that results from $N_{10}$ systems in bin 10, is subtracted from the $\psi\left(q^{*}\right)$ histogram. Then there are no systems left in bin 10, and the process may be repeated to obtain $N_{9}, \ldots, N_{1}$. The numbers $N_{i}$ form a histogram which represents the real $\phi(q)$ distribution, corrected for the behaviour of $P_{q^{\prime}}\left(q^{*}\right)$.

The scheme may be summarized and formalized in the matrix notation:

$$
\left(\begin{array}{c}
n_{1} \\
n_{2} \\
\vdots \\
n_{k}
\end{array}\right)=\left(\begin{array}{cccc}
a_{11} & a_{12} & \cdots & a_{1 k} \\
a_{21} & a_{22} & & a_{2 k} \\
\vdots & & \ddots & \vdots \\
a_{k 1} & a_{k 2} & \cdots & a_{k k}
\end{array}\right)\left(\begin{array}{c}
N_{1} \\
N_{2} \\
\vdots \\
N_{k}
\end{array}\right)
$$

The vectors $\mathbf{n}=\left(n_{1}, \ldots, n_{k}\right)$ and $\mathbf{N}=\left(N_{1}, \ldots, N_{k}\right)$ represent the histograms of the observed pseudo $q^{*}$-distribution $\psi\left(q^{*}\right)$ and the real distribution $\phi(q)$ respectively. The elements $a_{i j}$ of the matrix A represent the fractions

$$
\begin{cases}a_{i j}=\int_{q_{j-1}}^{q_{j}} P_{q^{\prime}=q_{i}}(q) d q, & \text { for } i \geq j, \text { and } \\ a_{i j}=0, & \text { for } i<j .\end{cases}
$$

If we represent (4.12) by

$$
\mathbf{n}=\mathrm{A} \mathrm{N}
$$

then the real $\phi(q)$ distribution, represented by vector $\mathbf{N}$, is found from the pseudo distribution $\psi\left(q^{*}\right)$, represented by vector $\mathbf{n}$, by the inverse relation:

$$
\mathbf{N}=\mathrm{A}^{-1} \mathbf{n}
$$

\subsection{Refining the method}

The paper by Halbwachs (1987) shows that the method works, but the results obtained with it are rather crude. This is due to the large $\Delta q=0.1$ interval that was chosen $(k=10)$, and to the fact that any uncertainties or 'noise' in the pseudo mass-ratio distribution $\psi\left(q^{*}\right)$ are amplified in the deconvolved real distribution $\phi(q)$. We will address both problems in this section, in an attempt to refine Halbwachs' method.

When we look at the vectors $\mathbf{n}$ and $\mathbf{N}$ again, we may notice that the normalized versions of these vectors $\mathbf{n} / N$ and $\mathbf{N} / N$ (where $N=\sum n_{i}=\sum N_{i}$ ) are good approximations of $\psi\left(q^{*}\right)$ and $\phi(q)$, since $\psi\left(q_{i}\right) \simeq n_{i} / N$ and $\phi\left(q_{i}\right) \simeq N_{i} / N$. The approximation becomes better as $\Delta q \rightarrow 0$ and $k \rightarrow \infty$. The histograms have then 
become continuous curves, which are exact representations of the distributions $\psi\left(q^{*}\right)$ and $\phi(q)$.

However, the number of spectroscopic binary systems for which we have data is limited, and hence it is difficult to obtain a continuous pseudo mass-ratio distribution $\psi\left(q^{*}\right)$. We may circumvent the problem by 'smoothing' the $\psi\left(q^{*}\right)$ histogram. The aim of the smoothing is to obtain a continuous $\psi\left(q^{*}\right)$ distribution from discrete data (from DAO8). The smoothing technique employed here smears every data point $q_{l}$, obtained from the Catalogue by applying Eq. (4.6), with a normal distribution $f\left(q_{l}\right)=$ $\left(\sigma_{q} \sqrt{2 \pi}\right)^{-1} \exp \left[-\left(q_{l}-q\right)^{2} / 2 \sigma_{q}^{2}\right]$. A smoothed version of $\psi\left(q^{*}\right)$ is then obtained by adding the $f\left(q_{l}\right)$ for all $l$ :

$$
\psi\left(q^{*}\right)=\frac{1}{L} \sum_{l=1}^{L} \frac{1}{\sigma_{q} \sqrt{2 \pi}} e^{-\left(q_{l}-q^{*}\right)^{2} / 2 \sigma_{q}^{2}},
$$

where $L$ is the number of systems in the sample. The amount of smoothing is controlled by varying the 'smoothing length' $\sigma_{q}$.

What amount of smoothing should we apply? With histograms, the error $\sigma_{\phi}$ in a distribution $\phi(x)$ depends on the bin width $\Delta x$ or the smoothing length $\sigma_{x}$. The two are related through a kind of 'uncertainty relation': $\sigma_{\phi} \propto \sigma_{x}^{-1}$. By varying the smoothing length $\sigma_{q}$, we are in control of the error $\sigma_{x}$ in $\phi(x)$. But whatever accuracy is gained in $\phi(x)$, is lost in $x$, since in order to lower $\sigma_{\phi}, \sigma_{x}$ has to be increased.

The error $\sigma_{\psi}$ in the smoothed histogram $\psi\left(q^{*}\right)$ may be determined by means of the Bootstrap technique. For a discussion of the Bootstrap and other resampling plans, see Efron (1982). For the samples of SBI and SBII systems in DAO8, it so turned out that a relative error of about $10 \%$ in $q$ (at $q=1$; hence a smoothing length $\sigma_{q}=0.1$ ), is cause for a relative uncertainty $\sigma_{\phi}$ in the reconstructed $\phi(q)$ of also $\approx 10 \%$.

\subsection{Checking the method: SBII systems}

The Eighth version of the DAO (Batten et al. 1989) was available in machine-readable form. This made it possible to computerize the whole process of: 1 . reading the data, 2. calculating the individual $q_{l}$ values, 3 . smoothing the data to obtain the pseudo mass-ratio distibution $\psi\left(q^{*}\right)$, and 4 . calculating the real mass-ratio distribution $\phi(q)$.

The individual $q_{l}$ were calculated employing Eq. (4.6). The primary masses $M_{1}$ were determined from the spectral-type-mass relation proposed by Trimble (1974). As indicated in the previous section, it was found that the pseudo mass ratio distribution $\psi\left(q^{*}\right)$ for the single-lined systems in DAO8 showed an error $\sigma_{\psi}<10 \%$ on average in $\psi\left(q^{*}\right)$ when the smoothing length was set to $\sigma_{q} \simeq 0.1$. This smoothing length implies that in the $q$-direction no better resolution was obtained than by Halbwachs, when he decided to represent the data in histograms with bin-width $\Delta q=0.1$. However, since the continuous representation of $\psi\left(q^{*}\right)$ allows the use of very narrow bins, the real $\phi(q)$ distribution is no longer described in terms of 10 discrete $q_{i}$ values, but also by a (almost) continuous curve.

It is because of the much higher sampling rate (we use $k=200$ ) that the estimate $\phi(q)$ of the real mass-ratio distribution can be determined with much greater precision $(10 \%)$ than was obtained by Halbwachs $(\approx 35 \%)$. The assumption that the systems 


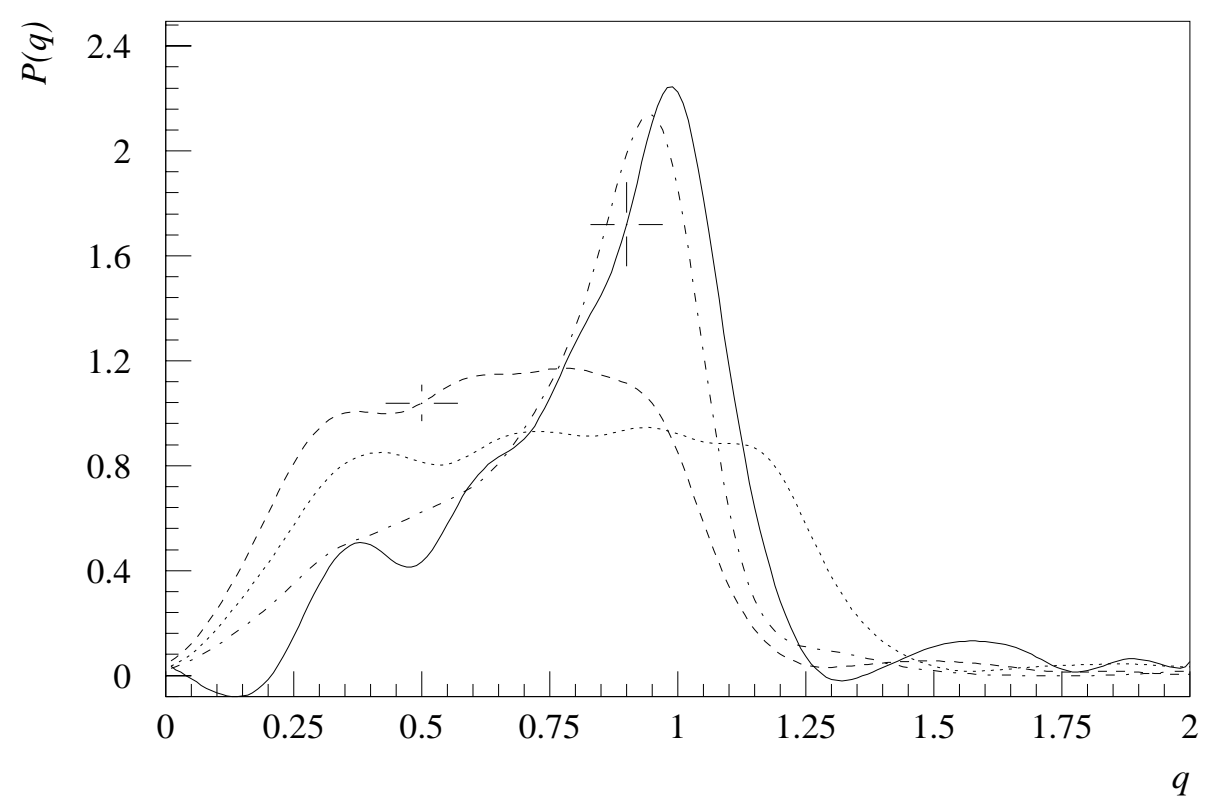

Figure 4.3: The deconvolution method of Eq. (4.13) applied to 366 SBII systems in DAO8 (Batten et al. 1989), assuming $P_{i}(i) d i=\sin i d i$. The dashed line represents the pseudo mass-ratio distribution, the solid line the reconstructed mass-ratio distribution, and the dash-dotted line the mass-ratio distribution as it is determined directly for SBII systems. The dotted line represents the mass-ratio distribution obtained with $\left\langle\sin ^{3} i\right\rangle=0.679$ in Eq. (4.2). The vertical error bars indicate $\sigma_{\psi}=0.07$ for the pseudo mass-ratio distribution $\psi\left(q^{*}\right)$, and $\sigma_{\phi}=0.16$ for the reconstructed real distribution $\phi(q)$. The horizontal error bars indicate the smoothing length for the histograms $\sigma_{q}=0.07$.

in the real $q$-distribution can only have discrete values $q_{i}$, introduces an error in the redistribution (or smearing) of these systems among the lower $q_{i}$ bins according to Eqs. (4.9) and (4.10). By making the bins narrower, this error becomes smaller. Also, the smoothing process removes the errors that arise from systems falling into the wrong bin due to errors in their $q$-values.

Following Halbwachs, the refined method was first applied to the double-lined spectroscopic binaries (SBII) in DAO8 to test its validity. In applying the method to the SBII systems, these were regarded as single-lined systems, and treated accordingly. However, since the mass ratios of SBII systems may be calculated directly from the observed radial velocity amplitudes: $q=M_{2} / M_{1}=K_{1} / K_{2}$, the SBII systems form a reference sample which allows us to test the method by comparing its results with the mass-ratio distribution that is determined directly.

Figure 4.3 shows the results of this exercise, using Eq. (4.9) to calculate matrix A. The sample comprises 366 SBII systems from DAO8 for which the spectral-type designations in the Catalogue contained no peculiarities.

The distributions were compared to eachother by means of the KolmogorovSmirnov test (Sachs 1981, Press et al. 1986). With a probability of $P_{\mathrm{KS}}=0.03$ that the distributions are the same, and maintaining a minimum level of confidence of $1 \%$ or $P_{\mathrm{KS}}=0.01$, the reconstructed mass-ratio distribution $\phi(q)$ compares rea- 


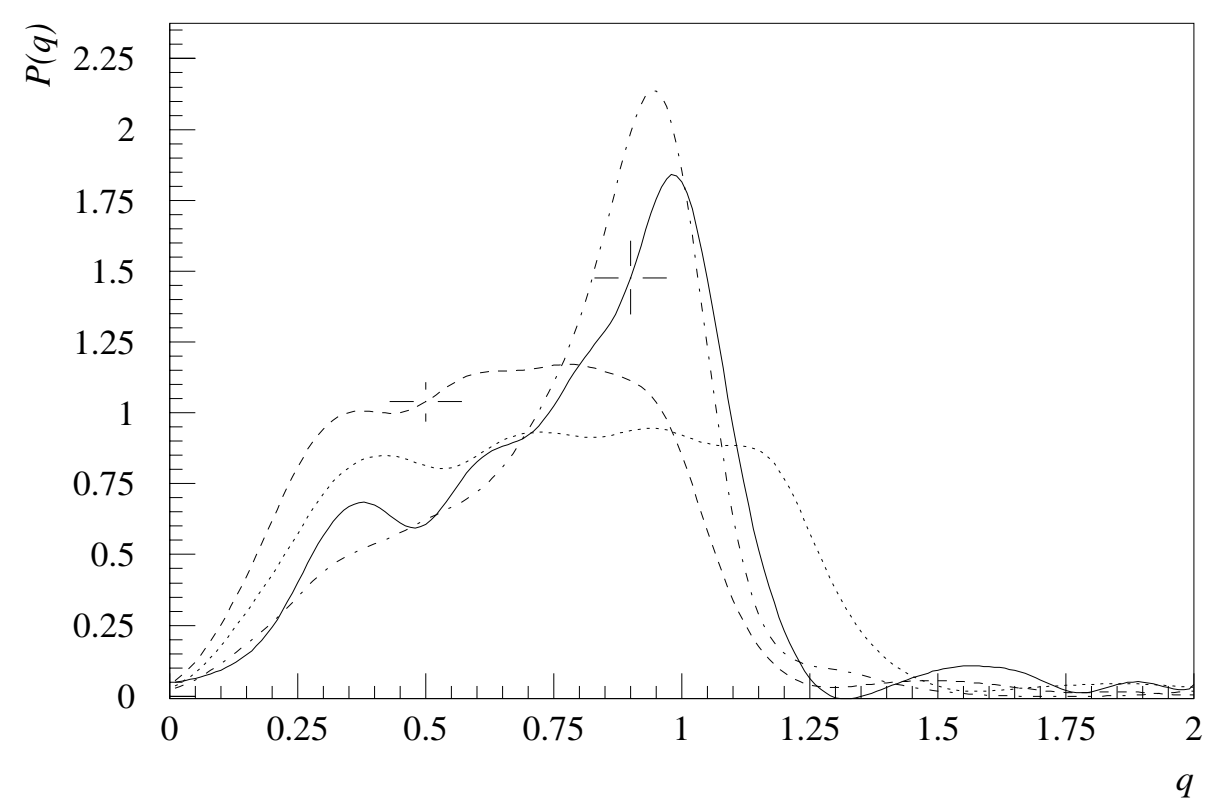

Figure 4.4: The deconvolution method of Eq. (4.13) applied to 366 SBII systems in DAO8 (Batten et al. 1989), assuming $P_{i}(i) d i=(4 / \pi) \sin ^{2} i d i$. The dashed line represents the pseudo mass-ratio distribution, the solid line the reconstructed mass-ratio distribution, and the dash-dotted line the mass-ratio distribution as it is determined directly for SBII systems. The dotted line represents the mass-ratio distribution obtained with $\left\langle\sin ^{3} i\right\rangle=0.679$ in Eq. (4.2). The vertical error bars indicate $\sigma_{\psi}=0.07$ for the pseudo mass-ratio distribution $\psi\left(q^{*}\right)$, and $\sigma_{\phi}=0.13$ for the reconstructed real distribution $\phi(q)$. The horizontal error bars indicate the smoothing length for the histograms $\sigma_{q}=0.07$.

sonably well to the distribution that was obtained directly. In the figure also the graph of $\phi(q)$ is drawn as it is obtained by calculating mass ratios from Eq. (4.2) with $\left\langle\sin ^{3} i\right\rangle=0.679$. Comparing this estimate of $\phi(q)$ with the directly obtained distribution by means of the KS-test yields $P_{\mathrm{KS}}=0.006$. Again maintaining a minimum level of confidence of $P_{\mathrm{KS}}=0.01$, the null-hypothesis must be rejected. Notice that in the case of the SBI systems, the $q$-distribution obtained with $\left\langle\sin ^{3} i\right\rangle=0.679$ is accepted as being representative of the real mass-ratio distribution.

Figure 4.4 shows the result of the deconvolution scheme using Eq. (4.10) to calculate matrix A. Real and reconstructed mass-ratio distribution now compare with a KS-test probability $P_{\mathrm{KS}}=0.85$, which is better than $P_{\mathrm{KS}}=0.03$ found above by assuming $P_{i}(i) d i=\sin i d i$.

The good comparison between real and reconstructed mass-ratio distribution confirms, at a higher level of confidence, the earlier findings of Halbwachs (1987) that the assumption of a random orientation of orbital planes of binary systems holds, at least for the SBII systems. 


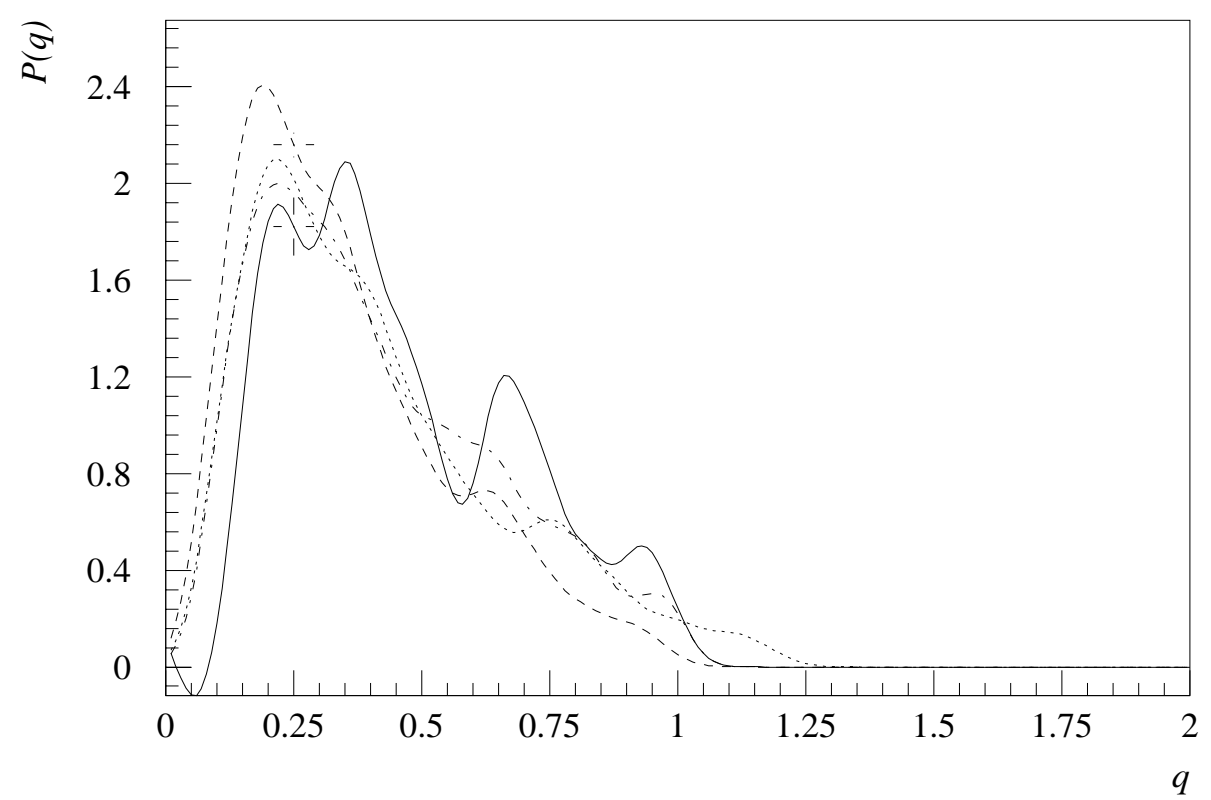

Figure 4.5: The deconvolution method (assuming $P_{i}(i) d i=\sin i d i$ ) applied to a synthetic sample of 1000 SBI systems that resulted from numerical simulations of the selection effects. The population of binary systems from which this sample resulted was generated with a random orientation of orbits in space. Solid line: reconstructed distribution $\phi(q)$. Dashed line: distribution of pseudo mass ratios $\psi\left(q^{*}\right)$. Dotted line: distribution obtained with $\left\langle\sin ^{3} i\right\rangle=0.679$ in Eq. (4.2). Dash-dotted line: real mass-ratio distribution. The vertical error bars indicate $\sigma_{\psi}=0.05$ for the pseudo mass-ratio distribution $\psi\left(q^{*}\right)$, and $\sigma_{\phi}=0.12$ for the reconstructed real distribution $\phi(q)$. The smoothing length for the histograms $\sigma_{q}=0.04$ is indicated by the horizontal error bars.

\subsection{Comparison with models}

For the purpose of investigating the selection effects that affect the observed massratio distributions of binary stars, a numerical model was developed that generates populations of binary stars and 'observes' these populations as they are affected by the selection effects implemented in the model. The model, and the results that were obtained with it, are described elswhere (Hogeveen 1991, this thesis Chapter 3).

The deconvolution method developed in this paper was also applied to the synthetic samples of SBI systems that resulted from the selection-effects simulations. Figure 4.5 shows the result of applying the deconvolution method to a synthetic sample of 1000 SBI systems, assuming $P_{i}(i) d i=\sin i d i$. The population of binaries in the model, from which this sample resulted, was generated with a random orientation of orbital planes in space. For the synthetic sample of SBI systems the original mass ratios, that were attributed to the systems when they were generated, are of course known. Contrary to the case of the real SBI systems, this allows us to plot the real mass-ratio distribution in the figure. According to the KS-test, the probability that real and reconstructed mass-ratio disribution are the same is $P_{\mathrm{KS}}=2.4 \cdot 10^{-5}$. This is a very poor comparison, and with a minimum confidence level of $P_{\mathrm{KS}}=0.01$ the hy- 


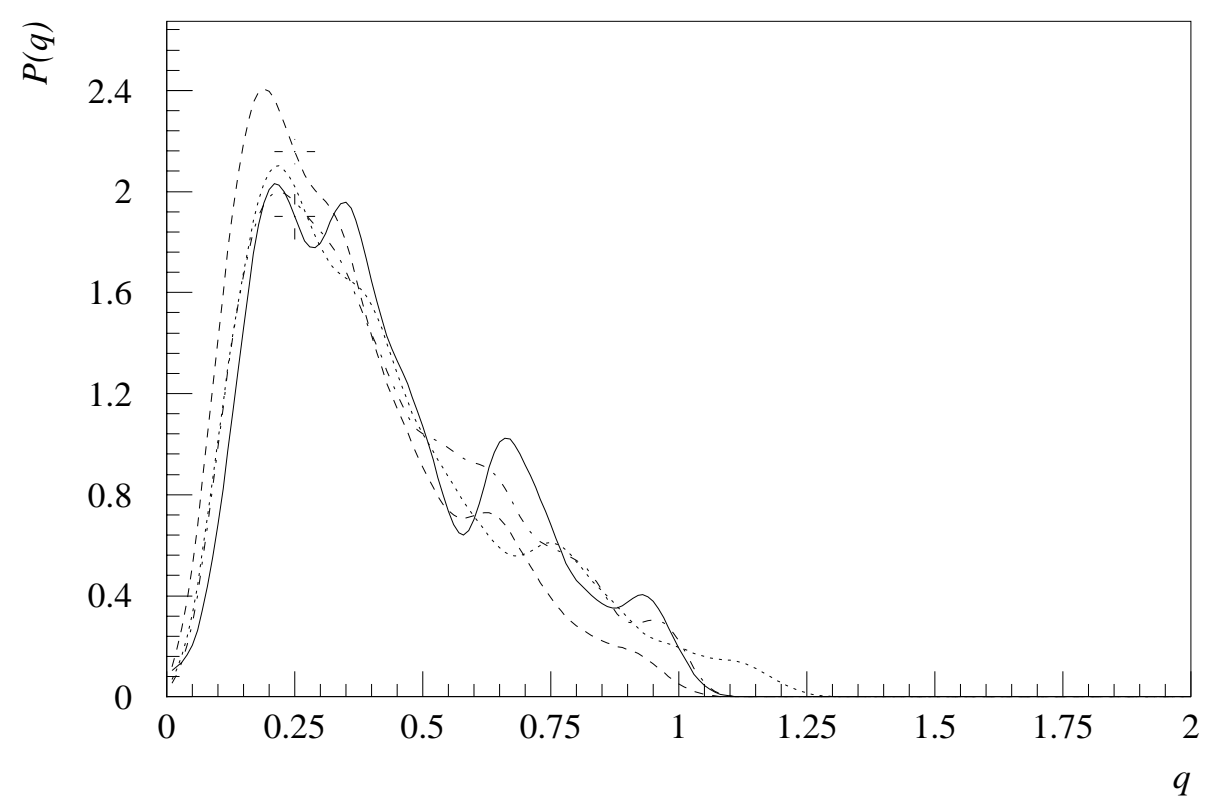

Figure 4.6: The deconvolution method (assuming $\left.P_{i}(i) d i=(4 / \pi) \sin ^{2} i d i\right)$ applied to a synthetic sample of 1000 SBI systems that resulted from numerical simulations of the selection effects. The population of binary systems from which this sample resulted was generated with a random orientation of orbits in space. Solid line: reconstructed distribution. Dashed line: distribution of pseudo mass ratios. Dotted line: distribution obtained with $\left\langle\sin ^{3} i\right\rangle=0.679$ in Eq. (4.2). Dash-dotted line: real mass-ratio distribution. The vertical error bars indicate $\sigma_{\psi}=0.05$ for the pseudo mass-ratio distribution $\psi\left(q^{*}\right)$, and $\sigma_{\phi}=0.09$ for the reconstructed real distribution $\phi(q)$. The smoothing length for the histograms $\sigma_{q}=0.04$ is indicated by the horizontal error bars.

pothesis that the distributions are the same must be rejected. The poor comparison is mainly caused by the undue shift in the maximum of the reconstructed distribution toward higher values of $q$. This shift is also observed when the reconstruction scheme is applied to real SBI systems (Trimble 1990). It is an artefact of the assumption that $P_{i}(i) d i=\sin i d i$.

The mass-ratio distribution determined with $\left\langle\sin ^{3} i\right\rangle=0.679$ compares to the real distribution with $P_{\mathrm{KS}}=0.97$. This probability is very high, and it means that in this case the method with the average value of $\sin ^{3} i$ provides a very good description of the real mass-ratio distribution.

The deconvolution scheme was applied to the same synthetic sample of 1000 SBI systems, assuming $P_{i}(i) d i=(4 / \pi) \sin ^{2} i d i$. The results are shown in Fig 4.6. Real and reconstructed mass-ratio distribution now compare with a KS-test probability $P_{\mathrm{KS}}=0.64$, which, with a minimum level of confidence of $P_{\mathrm{KS}}=0.01$, means that the statement that the distributions are the same may now be accepted. Although a comparison with $P_{\mathrm{KS}}=0.64$ is generally considered to be very good, it remains slightly less than the $P_{\mathrm{KS}}=0.97$ found for the comparison of real and 'average $\sin ^{3} i$ ' distribution. So, for the SBI systems, there is no benefit in applying the deconvolution scheme, which is more complex than the average $\sin ^{3} i$ method, and does not give 


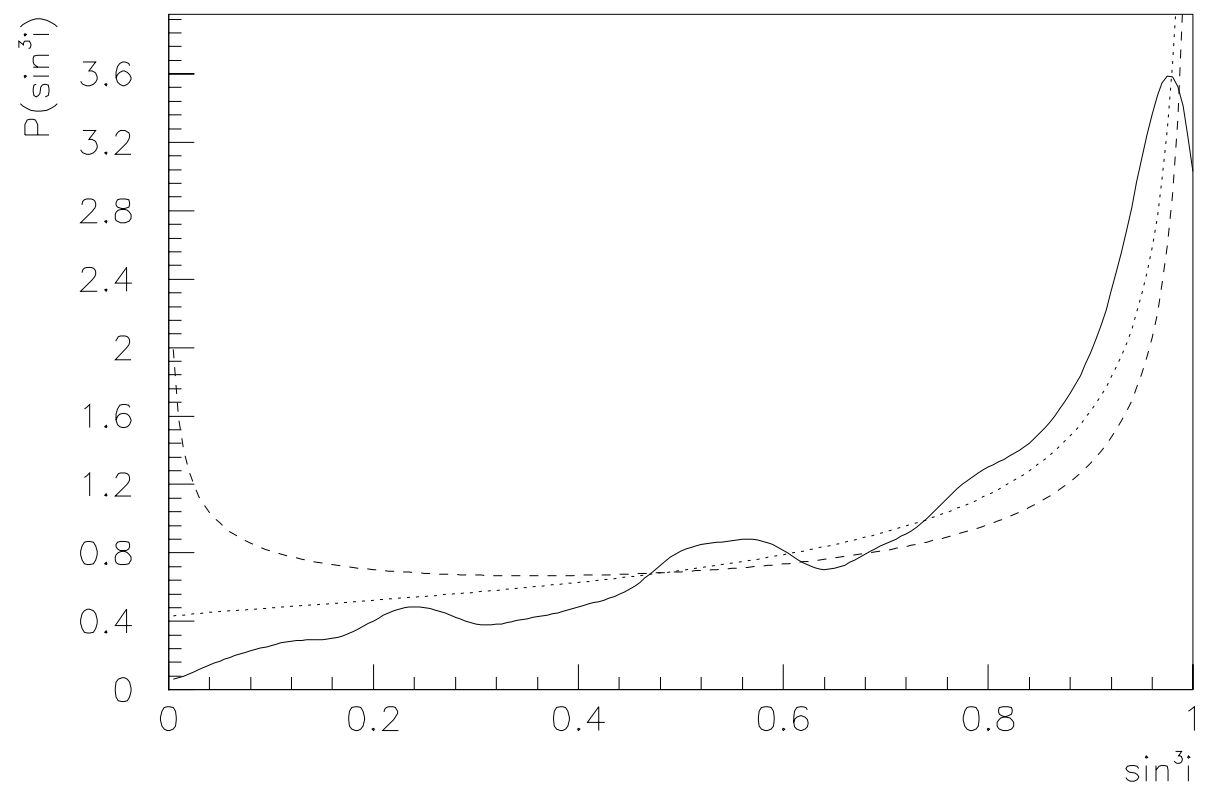

Figure 4.7: Observed distribution of $P\left(\sin ^{3} i\right) d \sin ^{3} i$ of the synthetic sample of SBI systems (solid line). The dashed line indicates the theoretical distribution of $\sin ^{3} i$ for orbital planes oriented according to $P_{i}(i) d i=\sin i d i$. The dotted line indicates the distribution $\operatorname{of}^{3} \sin ^{3} i$ for $P_{i}(i) d i=(4 / \pi) \sin ^{2} i d i$.

better results.

The results very much depend on the distribution of inclinations $P_{i}(i) d i$ that is used in the deconvolution scheme. For the systems in the synthetic populations, the inclinations of the orbits are known. Figure 4.7 shows the distribution of $\sin ^{3} i$ values of the SBI systems in the synthetic sample. It is obvious from the figure that $P_{i}(i) d i=(4 / \pi) \sin ^{2} i d i$ provides the better description of the 'observed' distribution of $\sin ^{3} i$ values of the SBI systems in the synthetic sample.

Following Halbwachs (1987), an attempt was made to derive the $P\left(\sin ^{3} i\right) d \sin ^{3} i$ distribution from observations in the following way. For the SBII systems in the DAO8 Catalogue it is possible to calculate $M_{1} \sin ^{3} i$. From a spectral-type-mass relation, an estimate of $M_{1}$ can be made. This is done for 319 main-sequence SBII systems in DAO8, using the spectral-type designations in the Catalogue. Figure 4.8 shows the result. The $P\left(\sin ^{3} i\right) d \sin ^{3} i$ distribution that was obtained is very crude (cf. error bars), but it does exhibit the expected trend. Again, the assumption of $P_{i}(i) d i=(4 / \pi) \sin ^{2} i d i$ provides the better description of the observed distribution.

It is very difficult to give a more accurate description of the observed $\sin ^{3} i$ distribution, because the departure from the theoretical $P\left(\sin ^{3} i\right) d \sin ^{3} i$ distribution (determined by a random distribution $P_{i}(i) d i=\sin i d i$ ) at low values of $\sin ^{3} i$ very much depends on the lowest observable radial-velocity amplitudes. All that can be established is that a distribution $P_{i}(i) d i=(4 / \pi) \sin ^{2} i d i$ generally provides a better description of the observed $P\left(\sin ^{3} i\right) d \sin ^{3} i$ distribution than the purely random $P_{i}(i) d i$. 


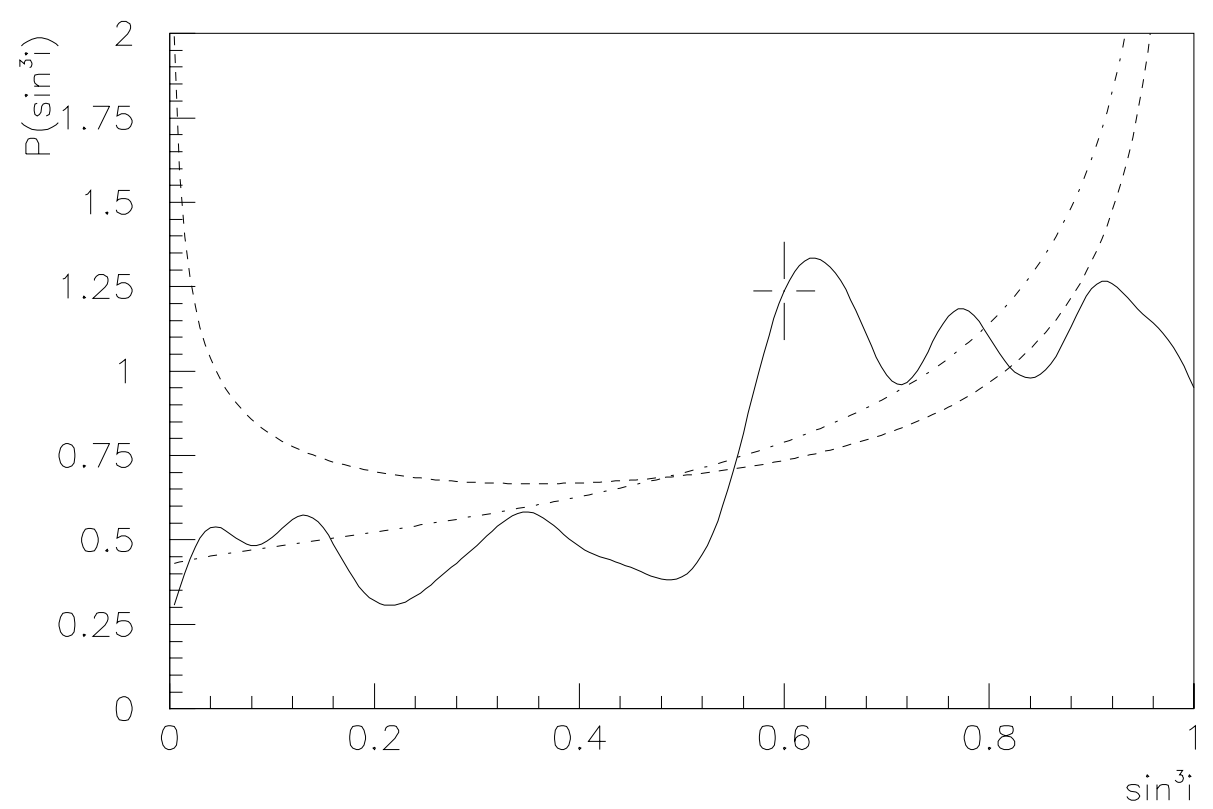

Figure 4.8: Distribution of $P\left(\sin ^{3} i\right) d \sin ^{3} i$ as derived for 319 main-sequence SBII systems from the DAO8 Catalogue (solid line). Dashed line: $P\left(\sin ^{3} i\right) d \sin ^{3} i$, assuming $P_{i}(i) d i=\sin i d i$. Dash-dotted line: $P\left(\sin ^{3} i\right) d \sin ^{3} i$, assuming $P_{i}(i) d i=(4 / \pi) \sin ^{2} i d i$.

\section{7 $\mathrm{SBI}$ systems}

The result of applying the method to the SBI systems in DAO8 is shown in figure 4.9. The sample comprises 688 SBI systems for which the spectral-type designations in the Catalogue contained no peculiarities.

In the figure also the mass-ratio distribution is shown which results from applying Eq. (4.2), assuming $\left\langle\sin ^{3} i\right\rangle=0.679$ after Trimble (1974) and Staniucha (1979). According to the KS-test, the probability that the reconstructed mass-ratio distribution and the distribution obtained by using an average value $\left\langle\sin ^{3} i\right\rangle=0.679$ are the same, is $P_{\mathrm{KS}}=0.5$. This is a very high probability $\left(P_{\mathrm{KS}} \gg 0.01\right)$, which is another indication that the deconvolution scheme does not produce results which are significantly better than the average $\sin ^{3} i$ method.

The similarity of the results for the synthetic sample of SBI systems (Fig. 4.5) and the DAO8 sample (Fig. 4.9), seems to confirm that the assumption, that orbits are oriented randomly in space, is correct.

\subsection{Conclusions}

Based on a scheme proposed by Halbwachs (1987), a refined method is developed to deconvolve the observed mass-ratio distribution of single-lined spectroscopic binary stars for the behaviour of the $P\left(\sin ^{3} i\right) d \sin ^{3} i$ distribution. The method is applied to a sample of SBII systems, for which the mass-ratio distribution is known from $q=M_{\text {sec }} / M_{\text {prim }}=K_{1} / K_{2}$. The reconstructed mass-ratio distribution is closer to the directly determined distribution when a distribution $P_{i}(i) d i=(4 / \pi) \sin ^{2} i d i$ is 


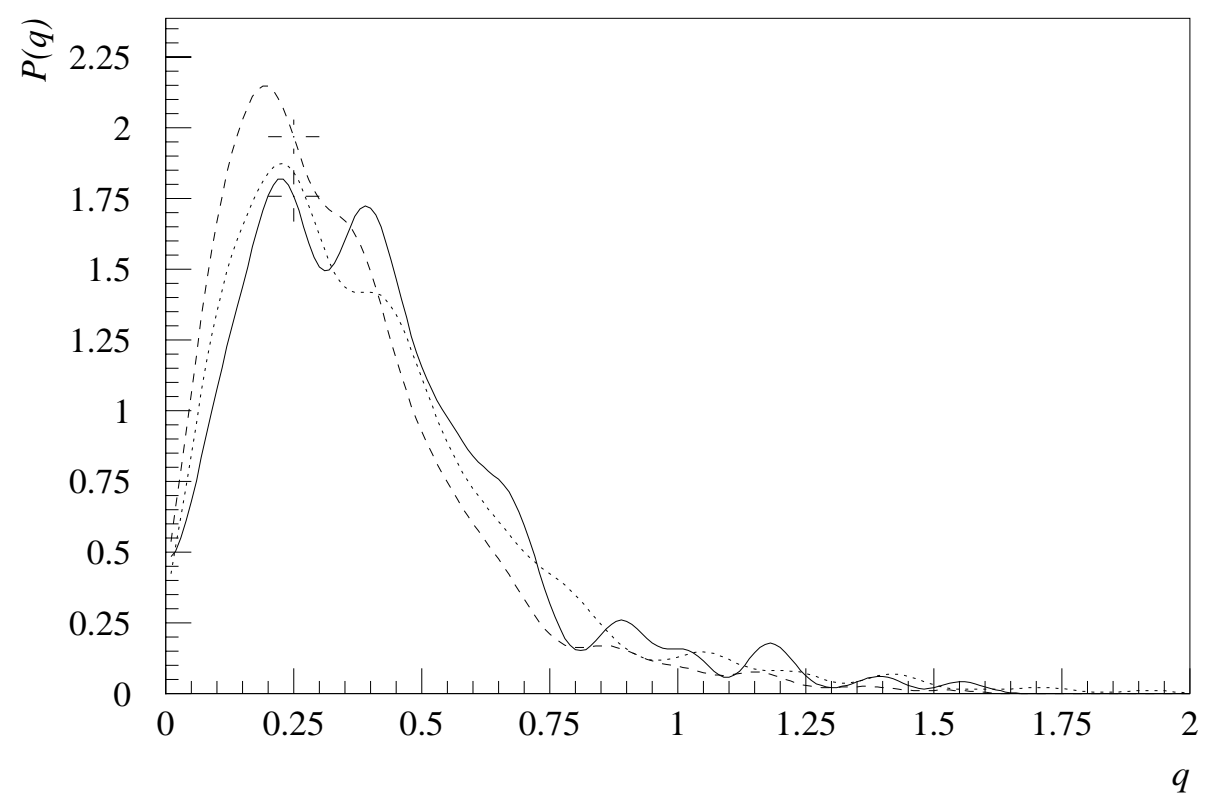

Figure 4.9: The deconvolution method of Eq. (4.13) applied to 688 SBI systems in DAO8 (Batten et al. 1989). The dashed line represents the pseudo mass-ratio distribution; the solid line the reconstructed mass-ratio distribution. The dotted line represents the mass-ratio distribution that is obtained by means of Eq. (4.2), assuming $\left\langle\sin ^{3} i\right\rangle=0.679$ after Trimble (1974) and Staniucha (1979). The vertical error bars indicate $\sigma_{\psi}=0.06$ for the pseudo mass-ratio distribution $\psi\left(q^{*}\right)$, and $\sigma_{\phi}=0.09$ for the reconstructed real distribution $\phi(q)$. The smoothing length for the histograms $\sigma_{q}=0.05$ is indicated by the horizontal error bars.

assumed for the orientation of orbital planes in space, rather than a purely random distribution $P_{i}(i) d i=\sin i d i$.

For a synthetic sample of SBI systems, generated by a numerical simulation of the selection effects, it is found that the deconvolution scheme provides results which are not acceptable (according to a statistical test) when a distribution $P_{i}(i) d i=\sin i d i$ is assumed. The results are acceptable according to the test when the distribution $P_{i}(i) d i=(4 / \pi) \sin ^{2} i d i$ is assumed. The distribution obtained for the synthetic sample of SBI systems by means of the deconvolution scheme is not better than the distribution obtained by using an average value $\left\langle\sin ^{3} i\right\rangle=0.679$ in Eq. (4.2).

For the synthetic sample of SBI systems, and for a sample of observed SBII systems from DAO8, it is shown that the assumption of $P_{i}(i) d i=(4 / \pi) \sin ^{2} i d i$ provides a better description of the observed distribution $P\left(\sin ^{3} i\right) d \sin ^{3} i$ than the assumption $P_{i}(i) d i=\sin i d i$.

The results obtained in this paper give a firmer basis to the decision of observers (Trimble 1974, Staniucha 1979) to use an average value $\left\langle\sin ^{3} i\right\rangle=0.679$ based on a distribution $P_{i}(i) d i=(4 / \pi) \sin ^{2} i d i$, rather than a value $\left\langle\sin ^{3} i\right\rangle=0.589$ based on $P_{i}(i) d i=\sin i d i$.

The shift in the maximum of the mass-ratio distribution of SBI systems to higher $q$-values, as observed by Trimble (1990) in applying Halbwachs' reconstruction 
scheme, is an artefact of the assumption of a purely random orientation of orbital planes in space. The shift disappears when the assumption $P_{i}(i) d i=(4 / \pi) \sin ^{2} i d i$ is entered in the deconvolution method.

When applied to the SBII systems in DAO8, the deconvolution scheme provides results which are compatible with the assumption of a random orientation of orbital planes in space for these SBII systems.

\section{Acknowledgements}

I wish to acknowledge stimulating discussions with Prof. Dr. E. P. J. van den Heuvel, and the valuable comments provided by Prof. Dr. J. van Paradijs and Dr. R. Wijers. I gratefully acknowledge the use of the computer facilities of, and support from, NikHEF-H (Netherlands Institute for Nuclear and High Energy Physics, section H) in Amsterdam. I wish to thank Drs. A. H. Batten and J. M. Fletcher of the Dominion Astronomical Observatory, Victoria, B.C., for providing me with a machine-readable version of the Eighth Catalogue of the Orbital Elements of Spectroscopic Binary Stars (Batten et al. 1989). My work is supported through the Netherlands Foundation for Research in Astronomy (Astron) by the Netherlands Foundation for the Advancement of Research (NWO), grant 782-371-026.

\section{References}

Abt, H. A. and Levy, S. G.: 1985, Astrophys. J., Suppl. Ser. 59, 229

Batten, A. H., Fletcher, J. M., and MacCarthy, D. G.: 1989, Eighth Catalogue of the Orbital Elements of Spectroscopic Binary Sytems, Dominion Astrophysical Observatory, Victoria, B.C., (DAO8)

Efron, B.: 1982, The Jackknife, the Bootstrap and Other Resampling Plans, Society for Industrial and Applied Mathematics, Philadelphia, Pennsylvania

Halbwachs, J. L.: 1987, Astron. Astrophys. 183, 234

Hogeveen, S. J.: 1991, Astrophys. Space Sci., submitted

Press, W. H., Flannery, B. P., Teukolsky, S. A., and Vetterling, W. T.: 1986, Numerical Recipes, Cambridge University Press, Cambridge

Sachs, L.: 1981, Applied Statistics, Springer-Verlag, New York

Staniucha, M.: 1979, Acta Astron. 29, 587

Trimble, V.: 1974, Astron. J. 79, 967

Trimble, V.: 1990, Mon. Not. R. Astron. Soc. 242, 79 